\title{
Safety of Cryo-Transbronchial Biopsy in Diffuse Lung Diseases: Analysis of Three Hundred Cases
}

\author{
Evgeni Gershman ${ }^{\mathrm{a}}$ Oren Fruchter ${ }^{\mathrm{a}}$ Fox Benjamin ${ }^{\mathrm{a}} \quad$ Abed Rahman Nader $^{\mathrm{a}}$ \\ Dror Rosengarten $^{\mathrm{a}}$ Victoria Rusanov ${ }^{\mathrm{a}}$ Ludmila Fridel $^{\mathrm{b}}$ Mordechai R. Kramer ${ }^{\mathrm{a}}$ \\ a Pulmonary Institute and ${ }^{b}$ Pathology Institute, Rabin Medical Center, Petah Tikva, Israel
}

\section{Key Words}

Bronchoscopy · Interventional technique - Cryobiopsy .

Transbronchial biopsy . Diffuse lung diseases $\cdot$ Safety

\begin{abstract}
Background: Transbronchial biopsy (TBB) which is performed with metal forceps (forceps TBB) has been accepted as a useful technique in establishing diagnoses of diffuse lung diseases (DLDs). The use of cryoprobes to obtain alveolar tissue (cryo-TBB) is a new method which is currently used by our institute as well as others with excellent results. $\mathbf{O b}$ jectives: To assess the safety of cryo-TBB compared with conventional forceps TBB. Methods: We performed a retrospective data evaluation of 300 consecutive patients who underwent cryo-TBB between January 2012 and April 2014 and compared them with historical cases treated with forceps TBB between 2010 and 2012. The results of both diagnostic modalities were compared based on pathological reports. The major complications (significant bleeding and pneumothorax) were compared, along with postprocedural hospitalization. Results: Pneumothorax was observed in 15 cases $(4.95 \%)$ treated with cryo-TBB versus 9 cases (3.15\%) treated with forceps TBB, with no significant difference $(p=$ 0.303). The insertion of a chest tube was necessary in $6(2 \%)$
\end{abstract}

and $4(1.3 \%)$ of the cases having undergone cryo-TBB or forceps TBB, respectively $(p=0.8)$. In the cryo-TBB group, bleeding was encountered in 16 cases (5.2\%), and it occurred in 13 cases (4.5\%) of the forceps TBB group, with no significant difference in rates $(p=0.706)$. Also, there was no significant difference in hospital admission rates between the groups [cryo-TBB: 10 (3.3\%); forceps TBB: 4 (1.44\%); $p=0.181$ ]. The safety profile of cryo- and forceps TBB remained the same even when stratified according to indications for TBB, i.e. immunocompromised hosts, patients after lung transplantation and those with DLDs. Conclusion: In patients with DLDs, cryo-TBB is as safe as forceps TBB.

(c) 2015 S. Karger AG, Basel

\section{Introduction}

Transbronchial biopsy (TBB; fig. 1) has generally been accepted as a useful technique in establishing a diagnosis of pulmonary infiltrates occurring in immunosuppressed patients [1-4] and lung transplant recipients [5-8], and it is commonly employed in the diagnostic evaluation of diffuse parenchymal lung disease [9-14]. Traditionally, TBB has been performed with metal forceps under fluoroscopy. Due to the small surface area of the samples ob-

\section{KARGER 125}

(c) 2015 S. Karger AG, Base

0025-7931/15/0901-0040\$39.50/0

E-Mail karger@karger.com

www.karger.com/res
Mordechai R. Kramer, MD, FCCP

Pulmonary Institute

Rabin Medical Center

Petah Tikva 4941492 (Israel)

E-Mail kramerm@ clalit.org.il 


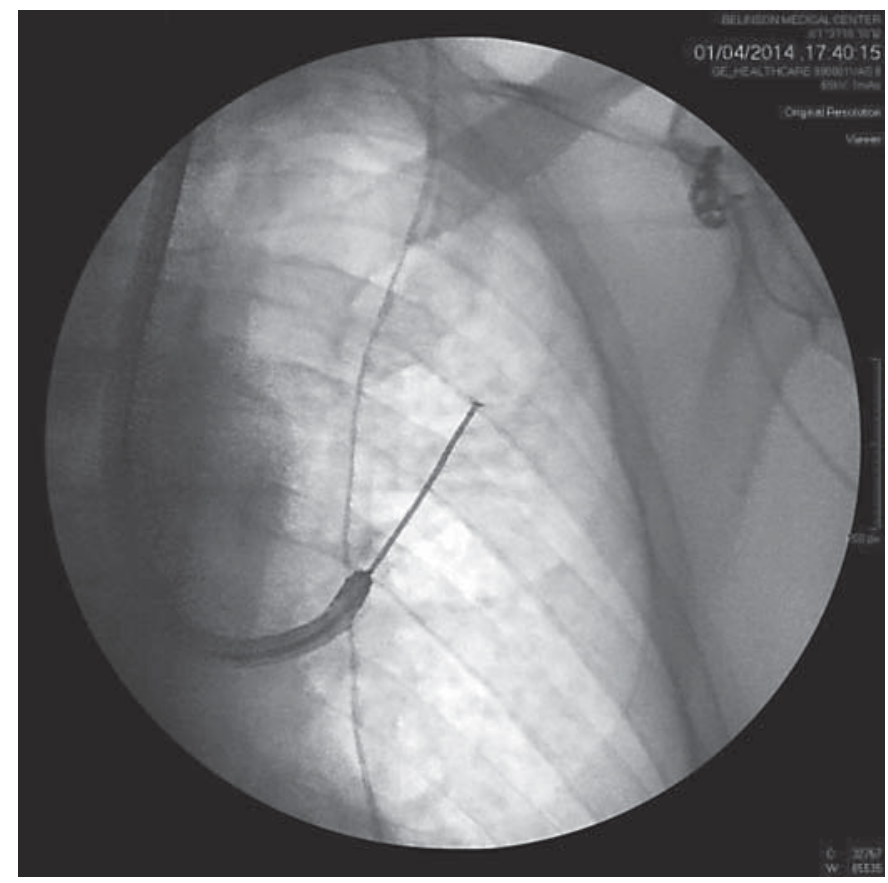

Fig. 1. Forceps TBB.

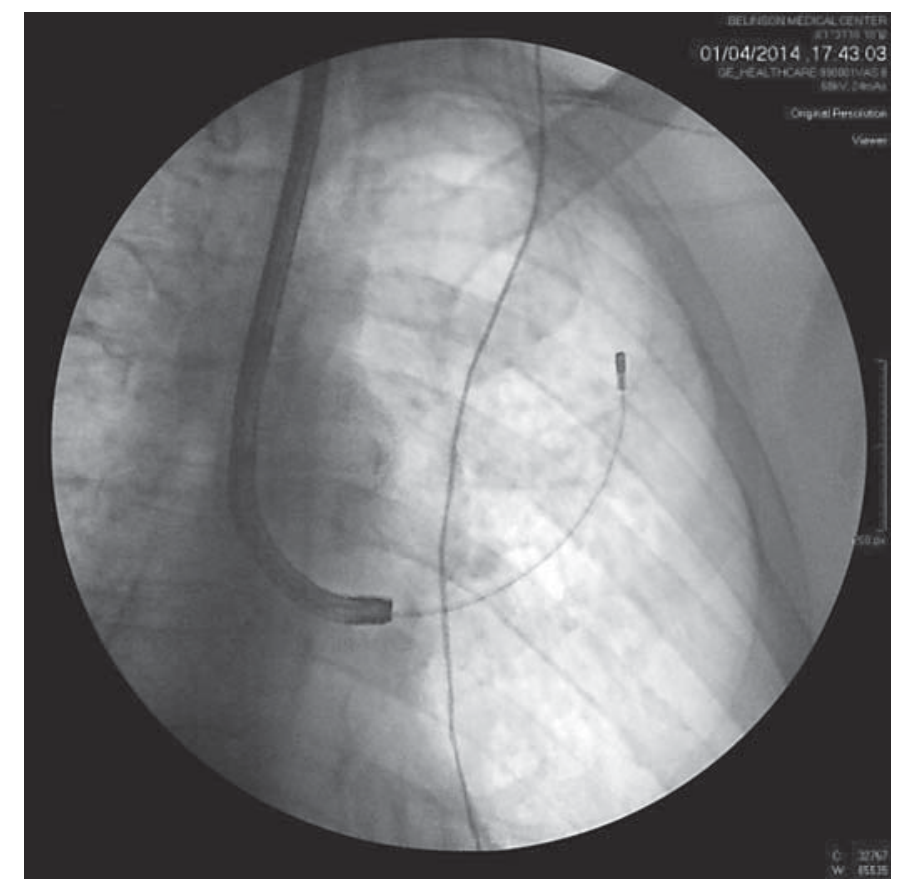

Fig. 2. Cryo-TBB. tained with a forceps, usually at least $6-8$ forceps biopsies are required to get a satisfactory amount of diagnostic material $[2,3,15,16]$. The main complications are pneumothorax and pulmonary bleeding [17]. The procedure occasionally may be associated with considerable morbidity and mortality in this fragile population [18].

Cryotherapy has been used in bronchoscopy for many years, and its main use is in the treatment and excision of endobronchial lesions, particularly in cases of bronchial obstruction [19-21]. The use of cryo-TBB (fig. 2) to obtain lung parenchyma has recently been introduced by several groups including our institute [22-27]. The surface area of lung tissue obtained by cryo-TBB has been shown to be significantly larger than that obtained by forceps, and the procedure has been shown to have an excellent safety profile $[22,27,28]$.

The aim of the current study was to assess the safety of cryo-TBB in comparison with conventional forceps TBB.

\section{Methods}

\section{Study Design}

We performed a retrospective data evaluation of 300 consecutive patients who underwent cryo-TBB between January 2012 and April 2014 or forceps TBB between 2010 and 2012. Patients in those periods of time were randomly allocated to either technique depending on the performer's choice. All bronchoscopies were performed by senior pulmonologist or residents under the supervision of seniors. Analysis of the data was approved by the institutional review board (No. 6668). Written informed consent for bronchoscopy and tissue sampling was obtained from all patients.

The data were gathered from network databases at the Rabin Medical Center, i.e. Broncho 2.0 bronchoscopy procedural information, PACS Carestream imaging data and OFEK 7BL data on patient follow-up and hospital admissions. The incidence rates of complications among the cryo-TBB cases were compared with those among 300 patients treated with conventional forceps TBB between January 2010 and January 2012. The two major complications (bleeding and pneumothorax) were documented and compared.

The intensity of bleeding was determined to be controllable by suction with a flexible bronchoscope without a formal scale. The necessity for other interventions routinely used at our institute for bleeding management, such as topical adrenaline instillation or the use of cold saline, was assessed. To rule out pneumothorax, a chest $\mathrm{X}$-ray was done within $2 \mathrm{~h}$ of the procedure. Since TBB is a semielective ambulatory procedure, hospital admission rates following the procedure were also documented.

\section{Bronchoscopy Procedure}

All procedures were carried out in the bronchoscopy suite. Local anesthesia was induced by application of $2 \%$ lidocaine to the oropharynx at the beginning of the procedure. Conscious sedation was started with intravenous injection of a bolus of 2-4 mg midazolam and $0.5-1 \mathrm{mg}$ alfentanil and was maintained with intermittent intravenous boluses of $1-3 \mathrm{mg}$ midazolam or $0.5 \mathrm{mg}$ alfentanil 
Table 1. Basic demographics of the patients

\begin{tabular}{lcll}
\hline $\begin{array}{l}\text { Parameter/ } \\
\text { lung pathology }\end{array}$ & Cryo-TBB & Forceps TBB & p value \\
\hline $\begin{array}{l}\text { Mean age, years } \\
\text { LTx }\end{array}$ & 54.48 & 52.5 & \\
ICH & 54.22 & 62.4 & 0.29 \\
DLD & 56.78 & 57.21 & 0.06 \\
Total & 56.14 & 55.83 & 0.84 \\
Gender (M/F), n & & & 0.8 \\
LTx & $86 / 60$ & $49 / 41$ & 0.59 \\
ICH & $14 / 4$ & $19 / 10$ & 0.52 \\
DLD & $39 / 50$ & $74 / 91$ & 0.9 \\
Total & $139 / 114$ & $142 / 142$ & 0.41 \\
\hline \multicolumn{2}{l}{ ICH = Immunocompromised host. } \\
\hline
\end{tabular}

or, in some cases, propofol (Diprivan) at doses of 20-30 mg, according to clinical judgment. Oxygen was insufflated continuously through a nasal cannula; spontaneous breathing was maintained during the entire procedure. Oxygen saturation, blood pressure, ECG and transcutaneous carbon dioxide partial pressure were continuously monitored.

The bronchopulmonary segment of the biopsy was the most affected segment, as determined prior to the procedure based on imaging studies of the chest. During flexible bronchoscopy, the bronchoscope (Olympus Exera) was introduced nasally or orally into the selected bronchus. A flexible cryoprobe (ERBE Elektromedizin $\mathrm{GmbH}$, Tübingen, Germany), $90 \mathrm{~cm}$ long and $2.4 \mathrm{~mm}$ in diameter, was introduced through the working channel of the bronchoscope under fluoroscopic guidance. Once brought into position, the probe was cooled with nitrous oxide to decrease the temperature of the probe's tip to $-89^{\circ} \mathrm{C}$ for approximately $4 \mathrm{~s}$, and then the entire bronchoscope was retracted with the frozen lung tissue attached to the probe's tip. The frozen specimen was thawed in saline and fixed in formalin.

Each patient underwent between 2 and 4 biopsies (the number of biopsies taken depended on the investigator's decision) using the cryoprobe as previously described. The specimens were fixed in $4 \%$ formalin and embedded in paraffin, and hematoxylin-eosin staining and PAS staining were conducted. Each slide was evaluated by an experienced pulmonary pathologist. Clinical features and the radiological pattern of the suggested lung disease were submitted to this pathologist. In addition to the suggested diagnosis, the pathological report included a description of the alveolated versus the bronchial tissue. The pathologist's report was stated to be 'nondiagnostic' if no conclusion could be made regarding the specimen analyzed.

\section{Statistical Analysis}

Statistical analyses were carried out by $\chi^{2}$ test for categorical variables, using Fisher's exact test (two-sided). The single noncategorical variable of age was compared using the $t$ test. A p value of $<0.05$ was considered significant. Data were analyzed using SPSS software v17.0 (IBM, Armonk, N.Y., USA) and SAS 9.4 (SAS Institute, Carey, N.C., USA).
Table 2. Safety profile results for cryo- and forceps TBB

\begin{tabular}{|c|c|c|c|}
\hline \multirow{2}{*}{$\begin{array}{l}\text { Complication/ } \\
\text { lung pathology }\end{array}$} & \multicolumn{2}{|l|}{ TBB } & \multirow{2}{*}{$\begin{array}{l}\mathrm{p} \\
\text { value }\end{array}$} \\
\hline & $\begin{array}{l}\text { cryo- } \\
(\mathrm{n}=300)\end{array}$ & $\begin{array}{l}\text { forceps } \\
(\mathrm{n}=300)\end{array}$ & \\
\hline \multicolumn{4}{|l|}{ Pneumothorax } \\
\hline \multicolumn{4}{|l|}{ Post-LTx } \\
\hline Total & $5 / 146(3.42)$ & $2 / 89(2.15)$ & 0.71 \\
\hline Chest tube & 1 & 1 & n.s. \\
\hline \multicolumn{4}{|l|}{$\mathrm{ICH}$} \\
\hline Total & $2 / 18(11.1)$ & 0 & 0.136 \\
\hline Chest tube & 2 & 0 & n.s. \\
\hline \multicolumn{4}{|l|}{ DLD } \\
\hline Total & $8 / 139(5.76)$ & $7 / 162(4.32)$ & 0.604 \\
\hline Chest tube & 3 & 3 & n.s. \\
\hline \multicolumn{4}{|l|}{ Total (\% TBB) } \\
\hline Total & $15 / 303(4.95)$ & $9 / 286(3.15)$ & 0.303 \\
\hline Chest tube & $6 / 303(2)$ & $4 / 286(1.33)$ & 0.803 \\
\hline \multicolumn{4}{|l|}{ Bleeding } \\
\hline Post-LTx & $6 / 146(4.11)$ & $2 / 89(2.15)$ & 0.489 \\
\hline $\mathrm{ICH}$ & $3 / 18(16.6)$ & $2 / 32(6.25)$ & 0.336 \\
\hline DLD & $7 / 139(5.04)$ & $9 / 167(5.38)$ & 1 \\
\hline Total (\% TBB) & $16 / 303(5.25)$ & $13 / 288(4.4)$ & 0.706 \\
\hline \multicolumn{4}{|l|}{ Hospitalization } \\
\hline Post-LTx & $3 / 146(2.05)$ & $1 / 87(1.15)$ & 1 \\
\hline $\mathrm{ICH}$ & $1 / 18(5.55)$ & 0 & 0.375 \\
\hline DLD & $6 / 139(4.31)$ & $3 / 160(1.88)$ & 0.313 \\
\hline Total (\% TBB) & $10 / 303(3.33)$ & $4 / 282(1.33)$ & 0.181 \\
\hline
\end{tabular}

Values are presented as $\mathrm{n}(\%) . \mathrm{ICH}=$ Immunocompromised host; n.s. = nonsignificant.

\section{Results}

\section{Patients}

The study group consisted of 300 patients who underwent cryo-TBB in our institute between January 2012 and April 2014. We compared them with 300 previous cases treated with forceps TBB between 2010 and January 2012. The preprocedural workup, premedication, facility, staff and postprocedural monitoring were identical for all patients. There were no differences in age or gender between the groups, as shown in table 1 .

\section{Complications}

The main complications of TBB were pneumothorax, bleeding and hospitalization after the procedure, as presented in table 2. Pneumothorax was observed in 15 cases $(4.95 \%)$ treated with cryo-TBB versus 9 cases $(3.15 \%)$ treated with forceps TBB, with no significant difference 
Table 3. Incidence of pneumothorax and bleeding in correlation with the number of cryobiopsies per session

\begin{tabular}{lllll}
\hline Complication & \multicolumn{2}{l}{ Number of biopsies } & p value \\
\cline { 2 - 4 } & 2 & 3 & 4 & \\
\hline Pneumothorax & $5(4.85 \%)$ & $4(4.4 \%)$ & $1(5.88 \%)$ & 0.98 \\
Bleeding & $5(4.85 \%)$ & $3(3.26 \%)$ & $1(5.88 \%)$ & 0.91 \\
\hline
\end{tabular}

$(\mathrm{p}=0.303)$. In the cryo-TBB group, bleeding was encountered in 16 patients (5.2\%), and it occurred in 13 patients (4.4\%) of the forceps TBB group. There was no significant difference in rates $(\mathrm{p}=0.706)$. Also, there was no significant difference in hospital admission rates between the groups, with $10(3.3 \%)$ and $4(1.44 \%)$ for the cryo-TBB and the forceps TBB group, respectively $(\mathrm{p}=0.181)$.

We further compared safety results between three categories of patients: those after a lung transplantation (post-LTx), immunocompromised hosts and cases with all other kinds of pathology [diffuselung diseases (DLDs)]. As presented in table 2 , in the cryo-TBB group pneumothorax was seen in $5(3.42 \%), 2(11.1 \%)$ and 8 cases (5.76\%) in the post-LTx, immunocompromised host and DLD categories, respectively. In the forceps TBB group, pneumothorax occurred in $2(2.25 \%), 0(0 \%)$ and 7 cases $(4.32 \%)$ in the post-LTx, immunocompromised host and DLD categories, respectively. As seen in table 2, there was no significant difference in rates of pneumothorax between the two TBB techniques when compared by the specific DLD categories $(\mathrm{p}=0.71, \mathrm{p}=0.136$ and $\mathrm{p}=0.604$, respectively).

We also analyzed the correlation of the incidence of pneumothorax with the need for chest tube drainage between the two main groups (cryo- vs. forceps TBB) and between the three categories mentioned above. The insertion of a chest tube was necessary in $6(2 \%)$ of the cryoTBB procedures versus 4 (1.3\%) of the forceps TBB procedures. The rates of chest tube drainage among the pneumothorax cases were 40 and $44.4 \%$ in the cryo- and forceps TBB groups, respectively $(\mathrm{p}=0.8)$.

In the cryo-TBB group, bleeding was encountered in 6 (4.11\%), $3(16.6 \%)$ and 7 cases (5.04\%) in the post-LTx, immunocompromised host and DLD categories, respectively (table 2). In the forceps TBB group, bleeding occurred in 2 (2.15\%), 2 (6.25\%) and 9 cases (5.38\%) in the post-LTx, immunocompromised host and DLD categories, respectively. As shown, there was no significant difference in procedure-related bleeding between the two
TBB techniques when compared by the lung pathological categories ( $\mathrm{p}=0.489, \mathrm{p}=0.336$ and $\mathrm{p}=1.0$, respectively). In both groups, none of the incidences of bleeding necessitated blood transfusion or other measures beyond local treatment with cold saline, adrenaline or Hexakapron during the bronchoscopy.

We also addressed the issue of whether an increase in biopsies performed during each session of cryo-TBB raised the incidence of complications. As presented in table 3 , there was a rise in the incidence of neither pneumothorax nor bleeding when more cryobiopsies were performed in a session ( $\mathrm{p}=0.98, \mathrm{p}=0.9$ and $\mathrm{p}=1$, respectively). Furthermore there was no statistically significant correlation between incidence rates of bleeding and pneumothorax in the cryo-TBB group (data not presented; $\mathrm{p}=0.32$ ).

Regarding the hospitalization rate, there was no need for hospitalization due to bleeding in any of the groups or categories. One lung transplant patient in the cryo-TBB group was hospitalized for several days as a consequence of sedation and anesthetic complications; he had no pneumothorax or bleeding. All other hospitalizations after TBB were due to pneumothorax. In the cryo-TBB group, the hospitalization rates were 3 (2.05\%), 1 (5.55\%) and $6(4.31 \%)$ in the post-LTx, immunocompromised host and DLD categories, respectively. In the forceps TBB group, the hospitalization rates were $1(1.15 \%), 0(0 \%)$ and $3(1.88 \%)$ in the post-LTx, immunocompromised host and DLD categories, respectively, as presented in table 2 . There was no difference in hospitalization rates between patients with any specific lung pathology $(\mathrm{p}=1.0$, $p=0.375$ and $p=0.313$, respectively) regardless of the TBB technique used.

\section{Discussion}

Acquiring tissue from diseased lung parenchyma is crucial for diagnosis and treatment in many circumstances. Several techniques have evolved over the years and are still employed in many centers; one of the most widely used is TBB. Cryo-TBB has been newly implemented in the diagnostic bronchological field; it has been taken up as a rather new modality and performed at our institute and many others.

In this study we compared the safety of cryo-TBB with that of forceps TBB. We also compared the TBB results for cryo- and forceps TBB in the categories based on lung pathology (post-LTx, immunocompromised hosts and DLDs). We found that the incidence of pneumothorax 
was $3-5 \%$, with no statistical difference between the two TBB techniques. Similar to results published in previous series regarding major complications of forceps TBB, the incidence rate of pneumothorax was 5\% and that of pulmonary hemorrhage was $2.8 \%$ [17].

Several cases of pneumothorax, after both forceps and cryo-TBB, resulted in hospitalization and the insertion of chest decompression tubes. The rates of chest tube insertions among all pneumothorax cases and the total number of procedures were similar, with no statistical difference between the two TBB techniques.

Our experience showed that all incidences of bleeding were controlled locally (cold saline, adrenaline or Hexakapron during bronchoscopy). None of the incidences of bleeding necessitated hospital admission, blood transfusion or other measures. No differences were found in hospitalization rates even when comparing between specific lung pathologies.

As previously mentioned, we did not use airway protection such as a laryngeal mask or formal intubation to facilitate bronchoscopic access, and we retrieved the bronchoscope with the cryoprobe en bloc after each biopsy. Although this reinstitution potentially causes epistaxis in the course of bronchoscopy, we did not encounter any increase in its incidence or any difference in the incidence of general airway bleeding. As presented in table 2 , the complication rates after cryo-TBB seem higher, yet there is no statistical difference. Studies with larger cohorts may present more complications, thus showing different results.

Several previous studies from our institute have shown excellent results regarding cryoprobe biopsies. Some patients from former studies were included in this study in order to emphasize the pathological results, aiming to evaluate only the safety of the procedure. Fruchter et al. [27] showed an excellent safety profile and histological results in a series of cryo-TBBs in lung-transplanted patients from our institute. In a retrospective study matching 40 transplanted patients who underwent cryoprobe biopsy with 40 transplanted patients who underwent forceps TBB, no bleeding or pneumothorax was encountered in the cryo-TBB study group.

In another series from our institute, Fruchter et al. [28] showed that in immunocompromised patients with pulmonary infiltrates, cryo-TBB provides clinically important diagnostic data with a low complication rate. In this series, no pneumothorax was observed, and only 1 patient suffered from bleeding, which was controlled with cold saline through the working channel of the bronchoscope.
Recently, we reported on the efficacy and safety of cryo-TBB in the diagnosis of interstitial lung diseases [29]. Only 3 patients (4\%) needed bleeding control by cold saline instillation, and 2 patients $(2.6 \%)$ had slight pneumothorax after the procedure, none of which required hospital admission; they resolved spontaneously within $72 \mathrm{~h}$ without any need for aspiration or chest tube drainage. It may be concluded that cryo-TBB is a safe and effective, minimally invasive modality for the diagnosis of interstitial lung disease that obviates the need for open lung biopsy for the vast majority of patients.

Previous studies have shown that the cryoprobe procedure resulted in obtaining larger tissue samples than with forceps TBB $[23,28,29]$. One can argue that having a potentially large defect in the lung parenchyma following cryobiopsy puts the patient at greater risk of bleeding and pneumothorax. However, in our experience, as presented in this study and in our previously published studies [27-29], the occurrence of pneumothorax and bleeding was as low as with forceps biopsies, presumably due to the hemostatic and sealing effects of cooling on the margins of the parenchymal defect $[28,30]$.

A prospective safety study by Yarmus et al. [30] showed excellent results of cryoprobe biopsies of lung transplants. The authors performed 10 forceps biopsies and 5 cryobiopsies during the same session on a single lung in a total of 21 cases. There was only 1 case of pneumothorax, and even though there was a high incidence of documented bleeding - practically in all cases - these bleedings were graded 0 or 1 , meaning traces of blood requiring suction for control at most.

Experience reported in a retrospective study by Kropski et al. [31] showed an excellent safety profile for cryoTBB in patients with suspected diffuse parenchymal lung disease. In that study, a total of 37 patients underwent cryoprobe biopsy for DLD or familial interstitial pneumonia. There was no pneumothorax or bleeding requiring additional intervention. One day after the biopsy, 1 patient was admitted to a hospital due to hemoptysis, which spontaneously resolved. Another patient was admitted to the ICU due to hypoxemia, which returned to baseline levels after $12 \mathrm{~h}$. In contrast to the practice at our institute, they used general anesthesia and tracheal intubation during bronchoscopy. Additionally, they took a total of 2 biopsies, each from a different segment of the lung, unlike our practice of taking 2-3 biopsies from 1 lung.

In a recent prospective study by Casoni et al. [32], the authors evaluated the safety, feasibility and diagnostic yield of cryo-TBB in fibrotic DLDs in 69 cases. Unlike our
Gershman/Fruchter/Benjamin/Nader/ Rosengarten/Rusanov/Fridel/Kramer 
method of intravenous sedation and flexible bronchoscopy, they used rigid tracheoscope intubation and heavy sedation during the procedure. To minimize hemorrhage, they used an inflated Fogarty catheter immediately after the biopsy, which was deflated in the absence of bleeding. Nonetheless, prolonged bleeding was seen in 1 case $(1.4 \%)$ despite the use of a Fogarty catheter. Even though they did not elaborate on how the bleeding was stopped, they stated that no double-lumen intubation or surgery was necessary. The rate of pneumothorax was relatively high, reaching $28 \%$ of cases (19 patients), compared with our study, which showed a rate between 0.9 and $13.3 \%$ depending on the patient category, with a total average of $4.87 \%$. The authors state that the risk of pneumothorax was greater when fragments of pleura were present, with no correlation to number or size of the specimens. In their series, 1 patient, who was HIV positive, suffered from pneumothorax and was treated with chest drainage and high-flow oxygen, which led to an exacerbation of idiopathic pulmonary fibrosis, diffuse alveolar damage and, eventually, death.

In all our experience in cryo-TBB from previous studies and the current study, several patients were treated in the ICU after pneumothorax, but no fatalities were registered. The difference in safety profile between our study and that published by Casoni et al. [32] might be related to a difference in procedural technique (previously explained) or due to a selection bias with Casoni et al., who performed cryo-TBB on specific fibrosing DLDs. Nota- bly, we did not compare the risk of complications with the size of the specimen retrieved, which may explain the difference in the rates of complications between the two studies.

In this study, we have presented the gathered experience of the first 300 cases of TBB performed by cryo-TBB on suspected DLD and lung transplant patients. To the best of our knowledge, this is the largest study evaluating the safety of cryo-TBB. The major limitation to this study lies in its retrospective nature. As such, the gradation and severity of bleedings are subject to the views of those who perform the TBB, with a random distribution of patients to either TBB procedures by the performers' choice.

We have shown that there was no difference in the safety profiles of the two TBB techniques regarding pneumothorax, bleeding or the need for hospitalization. In this study, our aim was to evaluate the safety of the cryo-TBB procedure; therefore, we neither analyzed nor presented the pathological results. As we showed in previous studies from our institute, the pathological specimens obtained with cryo-TBB were larger and without crush artifacts, thus providing better specimens for pathologists. In conclusion, for patients with DLDs, cryo-TBB is as safe as forceps TBB and yields better pathological results.

\section{Financial Disclosure and Conflicts of Interests}

None.

\section{References}

1 McCabe RE: Diagnosis of pulmonary infections in immunocompromised patients. Med Clin North Am 1988;72:1067-1089.

2 Puksa S, Hutcheon MA, Hyland RH: Usefulness of transbronchial biopsy in immunosuppressed patients with pulmonary infiltrates. Thorax 1983;38:146-150.

$>3$ Cazzadori A, Di Perri G, Todeschini G, et al: Transbronchial biopsy in the diagnosis of pulmonary infiltrates in immunocompromised patients. Chest 1995;107:101-106.

$\checkmark 4$ Jain P, Sandur S, Meli Y, et al: Role of flexible bronchoscopy in immunocompromised patients with lung infiltrates. Chest 2004;125: 712-722.

$\checkmark 5$ Hopkins PM, Aboyoun CL, Chhajed PN, et al: Prospective analysis of 1,235 transbronchial lung biopsies in lung transplant recipients. J Heart Lung Transplant 2002;21:1062-1067.

6 Chakinala MM, Ritter J, Gage BF, et al: Yield of surveillance bronchoscopy for acute rejection and lymphocytic bronchitis/bronchiol- itis after lung transplantation. J Heart Lung Transplant 2004;23:1396-1404.

7 Wahidi MM, Ernst A: The role of bronchoscopy in the management of lung transplant recipients. Respir Care Clin N Am 2004;10: 549-562.

$\checkmark 8$ Chhajed PN, Aboyoun C, Malouf MA, et al: Risk factors and management of bleeding associated with transbronchial lung biopsy in lung transplant recipients. J Heart Lung Transplant 2003;22:195-197.

9 Margaritopoulos GA, Wells AU: The role of transbronchial biopsy in the diagnosis of diffuse parenchymal lung diseases: con (in English, Portuguese). Rev Port Pneumol 2012;18: 61-63.

10 Casoni GL, Cordeiro CR Jr, Tomassetti S, Romagnoli M, Chilosi M, Cancellieri A, Gurioli C, Poletti V: The role of transbronchial biopsy in the diagnosis of diffuse parenchymal lung diseases: pro (in English, Portuguese). Rev Port Pneumol 2012;18:57-60.
1 Verbeken EK: Classifying interstitial lung diseases in a fractal lung: a morphologist's view 'anno Domini 2000'. Eur Respir J Suppl 2001; 32:107s-113s.

-12 Tomassetti S, Cavazza A, Colby TV, Ryu JH, Nanni O, Scarpi E, Tantalocco P, Buccioli M, Dubini A, Piciucchi S, Ravaglia C, Gurioli C, Casoni GL, Gurioli C, Romagnoli M, Poletti V: Transbronchial biopsy is useful in predicting UIP pattern. Respir Res 2012;13: 96.

13 Oliveira CC, Fabro AT, Ribeiro SM, Defaveri J, Capelozzi VL, Queluz TH, Yoo HH: Evaluation of the use of transbronchial biopsy in patients with clinical suspicion of interstitial lung disease. J Bras Pneumol 2011;37:168175 .

14 Leslie KO, Gruden JF, Parish JM, Scholand MB: Transbronchial biopsy interpretation in the patient with diffuse parenchymal lung disease. Arch Pathol Lab Med 2007;131:407423. 
15 Kupeli E, Akcay S, Ulubay G, et al: Diagnostic utility of flexible bronchoscopy in recipients of solid organ transplants. Transplant Proc 2011;43:543-546.

16 Ettinger NA: Invasive diagnostic approaches to pulmonary infiltrates. Semin Respir Infect 1993;8:168-176.

17 Pue CA, Pacht ER: Complications of fiberoptic bronchoscopy at a university hospital. Chest 1995; 107:430-432.

18 Picard E, Schlesinger Y, Goldberg S, et al: Fatal pneumococcal sepsis following flexible bronchoscopy in an immunocompromised infant. Pediatr Pulmonol 1998;25:390-392.

19 Hetzel J, Eberhardt R, Herth FJ, et al: Cryobiopsy increases the diagnostic yield of endobronchial biopsy: a multicentre trial. Eur Respir J 2012;39:685-690.

20 Aktas Z, Gunay E, Hoca NT, et al: Endobronchial cryobiopsy or forceps biopsy for lung cancer diagnosis. Ann Thorac Med 2010;5: 242-246.

21 Schumann C, Hetzel J, Babiak AJ, et al: Cryoprobe biopsy increases the diagnostic yield in endobronchial tumor lesions. Thorac Cardiovasc Surg 2010;140:417-421.

22 Griff S, Ammenwerth W, Schönfeld N, et al: Morphometrical analysis of transbronchial cryobiopsies. Diagn Pathol 2011;166:53.
23 Babiak A, Hetzel J, Krishna G, et al: Transbronchial cryobiopsy: a new tool for lung biopsies. Respiration 2009;78:203-208.

24 Schuhmann M, Bostanci K, Bugalho A, Warth A, Schnabel PA, Herth FJ, Eberhardt R: Endobronchial ultrasound-guided cryobiopsies in peripheral pulmonary lesions: a feasibility study. Eur Respir J 2014;43:233-239.

25 Franke KJ, Theegarten D, Hann von Weyhern C, et al: Prospective controlled animal study on biopsy sampling with new flexible cryoprobes versus forceps: evaluation of biopsy size, histological quality and bleeding risk. Respiration 2010;80:127-132.

26 Franke KJ, Szyrach M, Nilius G, et al: Experimental study on biopsy sampling using new flexible cryoprobes: influence of activation time, probe size, tissue consistency, and contact pressure of the probe on the size of the biopsy specimen. Lung 2009;187:253-259.

27 Fruchter O, Fridel L, Rosengarten D, Raviv Y, Rosanov V, Kramer MR: Transbronchial cryo-biopsy in lung transplantation patients: first report. Respirology 2013;18:669-673.
28 Fruchter O, Fridel L, Rosengarten D, Rahman NA, Kramer MR: Transbronchial cryobiopsy in immunocompromised patients with pulmonary infiltrates: a pilot study. Lung 2013; 191:619-624.

29 Fruchter O, Fridel L, El Raouf BA, AbdelRahman N, Rosengarten D, Kramer MR: Histological diagnosis of interstitial lung diseases by cryo- transbronchial biopsy. Respirology 2014; 19:683-688.

30 Yarmus L, Akulian J, Gilbert C, Illei P, Shah P, Merlo C, Orens J, Feller-Kopman D: Cryoprobe transbronchial lung biopsy in patients after lung transplantation: a pilot safety study. Chest 2013;143:621-626.

31 Kropski JA, Pritchett JM, Mason WR, Sivarajan L, Gleaves LA, Johnson JE, Lancaster LH, Lawson WE, Blackwell TS, Steele MP, Loyd JE, Rickman OB: Bronchoscopic cryobiopsy for the diagnosis of diffuse parenchymal lung disease. PLoS One 2013;8:e78674.

32 Casoni GL, Tomassetti S, Cavazza A, Colby TV, Dubini A, Ryu JH, Carretta E, Tantalocco P, Piciucchi S, Ravaglia C, Gurioli C, Romagnoli M, Gurioli C, Chilosi M, Poletti V: Transbronchial lung cryobiopsy in the diagnosis of fibrotic interstitial lung diseases. PLoS One 2014;9:e86716. 